\title{
Comparison of lipid profile in different grades of non-alcoholic fatty liver disease diagnosed on ultrasound
}

\author{
Dhumal Uttareshvar Mahaling ${ }^{1}$, Madole Mahesh Basavaraj ${ }^{2}$, Aher Jagdish Bika ${ }^{3}$ \\ ${ }^{1}$ Assistant Professor in Radiodiagnosis, Government Medical College, Latur. India \\ ${ }^{2}$ Assistant Professor in Biochemistry, Maharashtra Institute of Medical Science and Research, Medical College, Latur. India \\ ${ }^{3}$ Junior Resident in Radiodiagnosis, Government Medical College, Latur. India
}

\section{PEER REVIEW}

Peer reviewer

Dr. Arun Kumar, Associate Professor, Department of Biochemistry, Manipal College of Medical Sciences, Pokhara, Nepal

E-mail: arun732003@gmail.com

Tel: +977-981666 4537

Comments

Over all the paper is worth publishing. It is short and descriptive and written well to attract readers' attention. The use of ultrasonography is clinically and financially practical for the diagnosis of patients with NAFLD . Details on Page 912

\begin{abstract}
Objective: To detect and compare serum lipid abnormalities in patients diagnosed with different grades of non-alcoholic fatty liver on ultrasonography.

Methods: A total of 70 cases which included 30 males and 40 females, diagnosed as nonalcoholic fatty liver disease (NAFLD) on ultrasound were investigated with serum lipid profile. Then a comparison of lipid abnormalities between different grades of fatty liver diagnosed on ultrasound was done. $P$ value was calculated by using analysis of variance test (ANOVA) and $P$ value $<0.05$ was considered as statistically significant.

Results: Out of 70 cases which were diagnosed as NAFLD on ultrasonography, grade I NAFLD cases were $47.15 \%$, grade II were $42.85 \%$ and grade III were $10 \%$. The mean age of the patients was 49.14 years. Male to female ratio was $3: 4$. Serum triglycerides, total cholesterol, LDL and VLDL levels were raised in $67.14 \%, 45.71 \% 34.28 \%, 25.71 \%$ of cases respectively. Low serum HDL levels were seen in $62.85 \%$ of patients. On statistical analysis we found increasing grades of NAFLD were significantly associated with increasing values of total cholesterol ( $P$ value-0.001), LDL $(P$ value$0.000)$ and VLDL ( $P$ value-0.003) and decreasing HDL $(P$ value-0.000).

Conclusion: Most of the patients of NAFLD in India is asymptomatic, non-diabetic and non-hypertensive. Though liver biopsy is the gold standard method for diagnosis of NAFLD, Ultrasonography which is non-invasive, simple tool, can be used for the early detection of NAFLD in asymptomatic patients.
\end{abstract}

\section{KEYWORDS}

Non-alcoholic fatty liver disease (NAFLD), Ultrasonography, Lipid profile nonalcoholic steatohepatitis (NASH)

\section{Introduction}

The term nonalcoholic steatohepatitis (NASH)[1] was coined by Ludwig in 1980 to describe the biopsy findings in patients with steatohepatitis in the absence of significant alcohol consumption. NASH is part of spectrum of steatosis, known as non-alcoholic fatty liver disease (NAFLD), which ranges from simple steatosis(fatty change/deposition) to steatohepatitis with fibrosis or cirrhosis[2]. A NAFLD

*Corresponding author: Dr. Madole Mahesh Basavaraj, Assistant Professor in Biochemistry, M.I.M.S.R. Medical College, Latur, Maharashtra-413512, India.

E-mail: maheshmadole@yahoo.co.in

Tel: 9423345286

Foundation Project: Funded by the department of radiodiagnosis with approval by institutional ethics committee of G.R. Medical College, Gwalior, Madhya Pradesh. India with Grant no.-GRM/01/15/2009. classification system (grade 1 to grade 3) has been proposed that correlates certain histologic features with the longterm prognosis[2,3]. In this classification system: Grade I constitutes simple steatosis. Grade II is steatosis with lobular inflammation and ballooned hepatocytes. Grade III is steatosis, lobular inflammation, ballooned hepatocytes and mallory hyaline or fibrosis.

NAFLD are now being increasingly recognized as a major health burden. The prevalence of fatty liver in India has

\section{Article history:}

Received 29 Aug 2013

Received in revised form 10 Sep, 2nd revised form 16 Sep, 3rd revised form 20 Sep 2013

Accepted 12 Nov 2013

Available online 28 Nov 2013 
been shown to be as high as $15 \%-30 \%[4]$, which is similar to that reported from some of the western countries[5,6]. Earlier reports indicated that majority of cases of NAFLD are relatively mild and have a benign course. However, now it has been documented that number of these cases can progress to fibrosis, cirrhosis, liver failure and hepatocellular carcinoma and thus contributes to liver related mortality and morbidity[7,8].

Most patients with NAFLD have no symptoms or signs of liver disease at the time of diagnosis, although many patients report fatigue or malaise and a sensation of fullness or discomfort on the right side of the upper abdomen. Hepatomegaly is the only physical finding in most patients[9].

Liver biopsy is a sensitive method for diagnosis of NAFLD. However, liver biopsy is a painful and invasive procedure[10] with rare, but potentially life threatening complications like bleeding[11,12] and is prone to sampling errors[13,14]. In addition, given the numbers of patients with NAFLD, the use of liver biopsy is clinically and financially impractical.

To evaluate and cofirm the usefulness of ultrasonography for diagnosis of NAFLD, the present study aims to diagnose NAFLD non-invasively by ultrasound and to compare ultrasonographically diagnosed NAFLD with serum lipid profile.

\section{Material and methods}

\subsection{Study area and case study}

Between September 2009 and September 2010, a total of 70 patients which included 30 males and 40 females were evaluated sonographically for fatty liver in our department. All patients of the age more than 18 years diagnosed as nonalcoholic fatty liver by ultrasound were included in the study only after taking informed consent from the patient. The approval from institutional ethics committee was duly taken before start of this research. Patient with history of alcohol intake more than $30 \mathrm{~g} / \mathrm{d}$ in males and more than $20 \mathrm{~g} / \mathrm{d}$ in females were excluded from the study. All the ultrasound examinations were performed on ALOKA Prosound SSD-4000SV using 2.5-6 MHz and TOSHIBA Nemio30 US Scanners using 3-5 MHz.

Subjects were considered as cases if they have fatty liver according to the standard criteria accepted by the American Gastroenterology Association i.e., an increase in hepatic echogenicity as a reference, the presence of enhancement and lack of differentiation in the periportal intensity and the vascular wall due to great hyperechogenicity in the parenchyma. The degree of involvement will be standardised with a semi quantitative scale of the degree of hepatic involvement. The diagnosis of hepatic steatosis was made on the basis of characteristic sonographic features: increased echogenecity of liver; increased liver contrast compared to kidney; vascular blurring-mainly of portal veins; attenuation of echogenic level in deep seated area.

\subsection{Grading of non-alcoholic fatty liver on ultrasonograohy}

Grade I: Minimal diffuse increase in the fine echoes. Liver appears bright compared to the cortex of the kidney (Figure 1). Normal visualization of diaphragm and intahepatic vessel borders (Figure 2).

Grade II: Moderate diffuse increase in the fine echoes. Slightly impaired visualization of the intrahepatic vessels and diaphragm (Figure 3).

Grade III: Marked increase in the fine echoes. Poor or no visualisation of intrahepatic vessels and diaphragm and poor penetration of the posterior, segment of the right lobe of the liver (Figure 4).

All patients diagnosed as NAFLD on USG were investigated for serum lipid profile. Then, a relationship between NAFLD and serum lipid profile was compared.
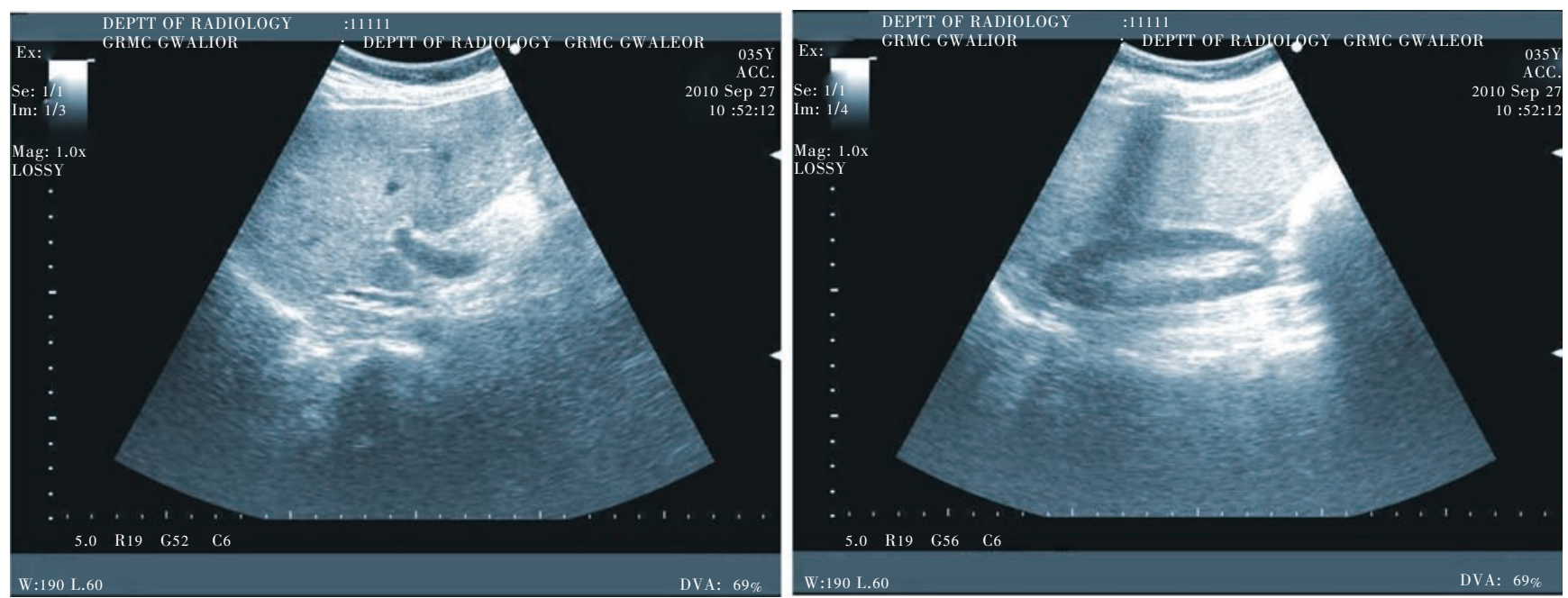

Figure 1. Sonogram showing increased liver echogenecity and liver-kidney contrast suggestive of grade I fatty liver. 


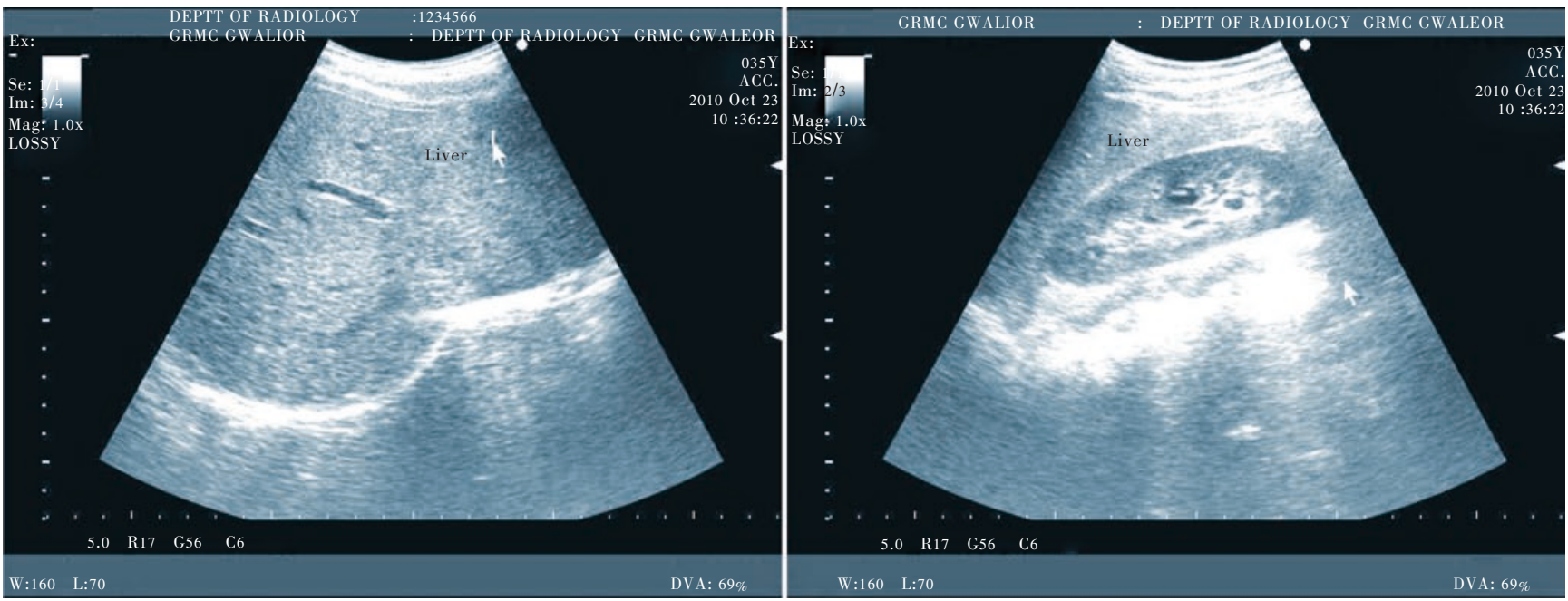

Figure 2. Sonogram showing example without fatty liver (normal patient).
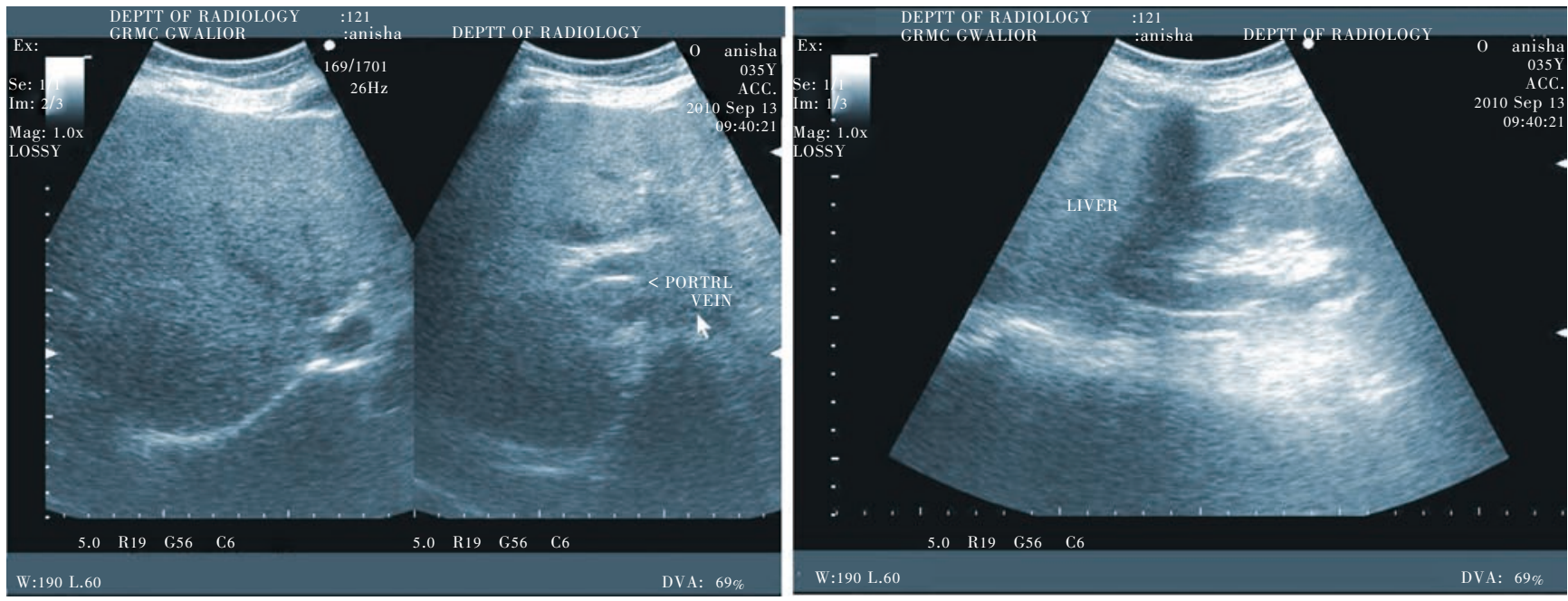

Figure 3. Sonogram showing increased liver echogenecity and liver-kidney contrast with blurring of peripheral portal vein margins and slight deep attenuation suggestive of grade II fatty liver.

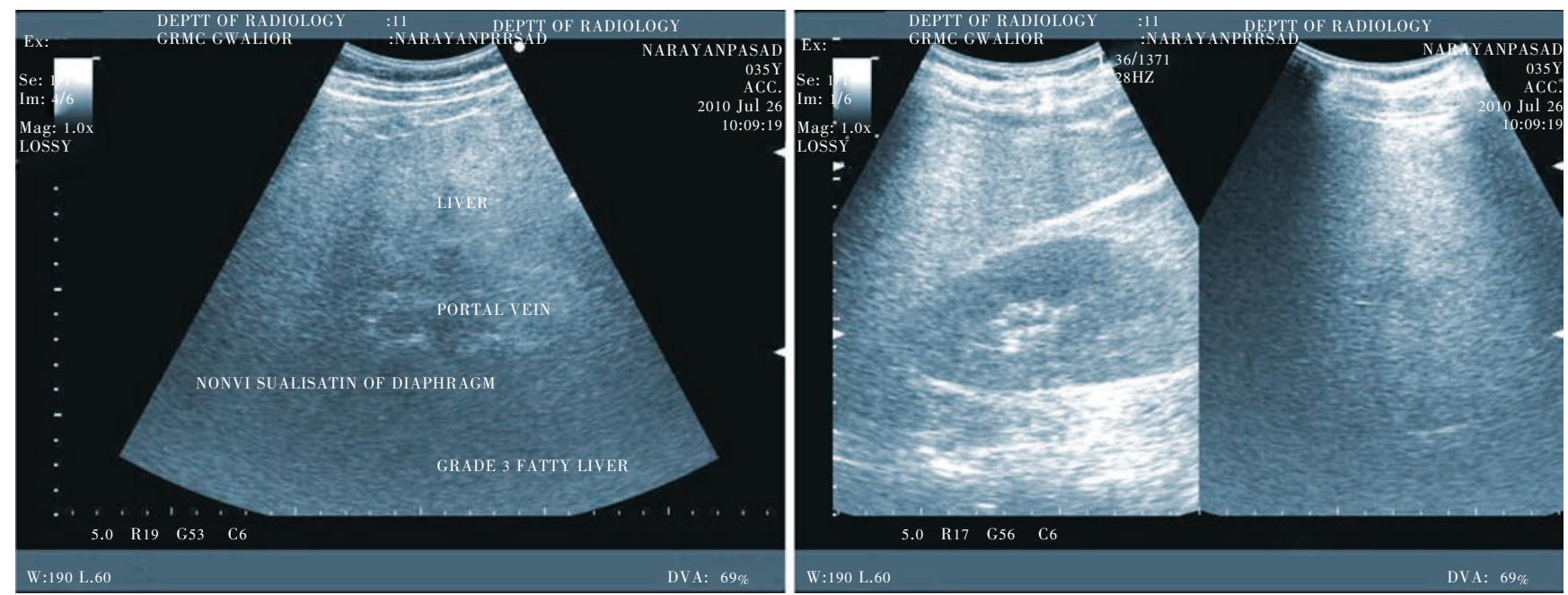

Figure 4. Sonogram showing increased liver echogenecity and liver-kidney contrast with nonvisualisation of portal vein margins and nonvisualisation of diaphragm and posterior part due to deep attenuation of ultrasound suggestive of grade III fatty liver.

\subsection{Statistical analysis}

The results were recorded on Microsoft excel 2007 sheet using windows XP. Mean values, standard deviation, charts were calculated using Microsoft excel sheet. $P$ value was calculated by using Analysis of variance test (ANOVA) and $P$ value $<0.05$ was considered statistically significant. 


\section{Results}

A total of 70 ultrasonographically diagnosed NAFLD cases were included in the study.

Table 1 shows the mean age of the patients was 49.14 years. Mean age in males was 49.06 years and in females was 49.20 years. Largest group of patients belonged to the fourth and fifth decades. Male to female ratio was 3:4. On ultrasonography, NAFLD was grade I in $47.15 \%$, grade II in $42.85 \%$ and grade III in $10 \%$. .

Table 1

Showing age distribution of NAFLD patients.

\begin{tabular}{|c|c|c|c|}
\hline Age group (years) & Grade I & Grade II & Grade III \\
\hline $18-27$ & 0 & 1 & 0 \\
\hline $28-37$ & 6 & 4 & 0 \\
\hline $38-47$ & 6 & 9 & 3 \\
\hline $48-57$ & 14 & 8 & 3 \\
\hline $58 \&$ above & 7 & 8 & 1 \\
\hline Total & 33 & 20 & 7 \\
\hline percentage & $47.15 \%$ & $42.85 \%$ & $10 \%$ \\
\hline
\end{tabular}

In the study group the mean age of the patients was $49.14_{+} 9.65$ years (range 25-66 years). The majority of patients were in the 48-57 years age group $25(35.71 \%)$ followed by $38-47$ years group $18(25.71 \%), 16$ $(22.85 \%)$ in 58 years and above and $11(15.71 \%)$ in below 37 years age group. Grade I NAFLD patients were $47.15 \%$, grade II were $42.85 \%$ and grade III were $10 \%$.

Table 2 shows 24 of 70 patients were asymptomatic, remaining 46 patients were symptomatic. Upper abdominal pain and fatigue was present in $55.71 \%$ and $52.85 \%$ patients respectively.

Table 2

Showing signs and symptoms in NAFLD patients.

\begin{tabular}{lccccc}
\hline $\begin{array}{l}\text { Signs and } \\
\text { symptoms }\end{array}$ & Grade I & Grade II & Grade III & Total & Percentage \\
\hline Abdominal pain & 16 & 19 & 4 & 39 & $55.71 \%$ \\
Fatigue & 17 & 16 & 4 & 37 & $52.85 \%$ \\
Malaise & 5 & 11 & 1 & 17 & $24.28 \%$ \\
Hepatomegaly & 6 & 3 & 1 & 10 & $14.28 \%$ \\
Asymptomatic & 12 & 10 & 2 & 24 & $34.28 \%$ \\
\hline
\end{tabular}

Out of 70 patients included in the study 24 (34.28\%) were asymptomatic. The symptoms and signs were elicited in the remaining $46(65.71 \%)$ patients. Among the symptomatic patients, abdominal pain was reported in $39(55.71 \%)$ patients, fatigue in $37(52.85 \%)$, malaise in 17 $(24.28 \%)$ and hepatomegaly in $10(14.28 \%)$ patients.

Table 3 shows serum triglycerides, total cholesterol, LDL and VLDL levels were raised in $67.14 \%, 45.71 \%, 34.28 \%, 25.71 \%$ of patients respectively. Low serum HDL levels were seen in $62.85 \%$ of patients.

Table 4 shows comparison of lipid changes in different grades of NAFLD by statistical analysis using Analysis of Variance Test (ANOVA). $P$ values $<0.05$ were considered as significant. It was observed that increasing grades of NAFLD were significantly associated with increasing levels of serum total cholesterol $(P$ value-0.001), LDL $(P$ value-0.000) and VLDL $(P$ value-0.003) and decreasing HDL $(P$ value0.000). No significant association was found between serum triglyceride levels $(P$ value-0.05) and increasing grades of sonographically diagnosed NAFLD.

Table 3

Distribution of patients showing abnormal serum lipid profile in NAFLD.

\begin{tabular}{lcccccccccc}
\hline Ultrasound Grades & \multicolumn{1}{c}{ Grade I } & \multicolumn{2}{c}{ Grade II } & \multicolumn{2}{c}{ Grade III } & \multicolumn{3}{c}{ Total } & \multicolumn{2}{c}{ Total Percentage $(\%)$} \\
\hline Serum lipid profile $(\mathrm{mg} / \mathrm{dL})$ & $\mathrm{N}$ & $\mathrm{A}$ & $\mathrm{N}$ & $\mathrm{A}$ & $\mathrm{N}$ & $\mathrm{A}$ & $\mathrm{N}$ & $\mathrm{A}$ & $\mathrm{N}$ & $\mathrm{A}$ \\
Triglyceride $(\mathrm{N}<150 \mathrm{mg} / \mathrm{dL})$ & 18 & 15 & 5 & 25 & 0 & 7 & 23 & 47 & 32.86 & 67.14 \\
Total cholesterol $(\mathrm{N}<200 \mathrm{mg} / \mathrm{dL})$ & 23 & 10 & 13 & 17 & 2 & 5 & 38 & 32 & 54.29 & 45.71 \\
HDL $(>40 \mathrm{mg} / \mathrm{dl}$ in female $\&>50 \mathrm{mg} / \mathrm{dL}$ in male) & 18 & 15 & 8 & 22 & 0 & 7 & 26 & 44 & 37.15 & 62.85 \\
LDL $(\mathrm{N}<130 \mathrm{mg} / \mathrm{dL})$ & 26 & 7 & 17 & 13 & 3 & 4 & 46 & 24 & 65.72 & 34.28 \\
VLDL $(\mathrm{N}-12-30 \mathrm{mg} / \mathrm{dL})$ & 26 & 7 & 22 & 8 & 4 & 3 & 52 & 18 & 74.29 & 25.71 \\
\hline
\end{tabular}

$\mathrm{N}-$ Normal, A-Abnormal

Serum triglycerides, total cholesterol, LDL and VLDL levels were raised in 67.14\%,45.71\% $34.28 \%, 25.71 \%$ of patients respectively. Low serum HDL levels were seen in $62.85 \%$ of patients.

Table 4

Comparison between NAFLD and Serum Lipid Profile

\begin{tabular}{lccccccc}
\hline Ultrasound Grades & \multicolumn{2}{c}{ Grade I } & \multicolumn{2}{c}{ Grade II } & \multicolumn{2}{c}{ Grade III } \\
\hline Serum Lipid Profile $(\mathrm{mg} / \mathrm{dl})$ & Mean & Standard Deviation & Mean & Standard Deviation & Mean & Standard Deviation & $P$ value \\
\hline Triglyceride & 162.7 & 52.49 & 220.53 & 119.41 & 276.71 & 119.76 & 0.05 \\
Total Cholesterol & 185.20 & 36.50 & 214.29 & 47.68 & 251.40 & 53.91 & 0.001 \\
HDL & 45.38 & 5.57 & 41.61 & 4.81 & 32.71 & 3.63 & 0.000 \\
LDL & 104.88 & 25.18 & 124.72 & 30.11 & 155.88 & 44.45 & 0.000 \\
VLDL & 25.55 & 5.77 & 27.75 & 3.70 & 35.42 & 16.42 & 0.003 \\
\hline
\end{tabular}

On statistical analysis using Analysis of Variance Test (ANOVA), comparison of lipid changes in different grades of NAFLD was made and $P$ values $<0.05$ was considered significant. It was observed that increasing grades of NAFLD were significantly associated with increasing levels of serum total cholesterol $(P$ value-0.001), LDL $(P$ value-0.000) and VLDL $(P$ value-0.003) and decreasing HDL $(P$ value-0.000). No significant association was found between serum triglyceride levels $(P$ value -0.05$)$ and increasing grades of sonographically diagnosed NAFLD. 


\section{Discussion}

NAFLD has emerged as the most common liver disease in the 'Western' economies. Prevalence of nonalcoholic fatty liver disease is rising in the Asia-Pacific region as the society becomes affluent and traditional lifestyles change (increasing fat in the diet, less physical activity, increasing prevalence of type 2 diabetes). NAFLD occurs in approximately $20 \%$ obese and 5\% overweight subjects. A 2.6 fold increase in prevalence of NAFLD was found when it occurred in association with type 2 diabetes[2]. It has been estimated that by 2020 number of people having type 2 diabetes will reach 100 million, 60\% of whom will live in Asia. NAFLD appears to be common in some ethnic groups like Philipinos, Indians and aboriginals of Australia/Malaysia. Thus, NAFLD is not a western disease. NAFLD can cause end stage liver disease including some cases of 'cryptogenic cirrhosis' and has been proposed to lead to hepatocellular carcinoma[8].

NAFLD has traditionally been described as a disease occurring predominantly in female patients who are obese, diabetic and hypertensive. Very few ultrasound based studies have been reported from India in NAFLD patients. In India, NAFLD occurs predominantly in men and majority of these patients are nonobese, non-diabetic and non-hypertensive. This demographic profile differs from that reported in the West.

The clinical and histological criteria for diagnosis of NASH have been described but NASH as a clinicopathological entity is still evolving. We undertook this study with the aim of diagnosing fatty liver on ultrasound in non-alcoholic patients presenting to radiodiagnosis department who are asymptomatic or with symptoms like abdominal pain, fatigue, malaise, etc.

In our study, age of the patients ranged from 25 years to 66 years with a mean age of $(49.14+9.65)$ years. Mean age in females was 49.20 years (range 30-66 years) and mean age in males was 49.06 years (range 25-65 years). In Indian studies mean age was reported to be $(42.90+10.54)$ years by Roli Agarwal et al[15], 55.4 years by Amarapurkar et al[16]. Most of the western studies have reported the mean age of NAFLD between 41-45 years. In our cases NAFLD most commonly seen in the fourth and fifth decade, this is about a 5-10 years elder than what has been reported from other countries.

Most of the patients with NAFLD are asymptomatic. The disease is discovered either incidentally during routine laboratory examination or when the patient is investigated for conditions like hypertension, diabetes or obesity. In our study group $24(34.28 \%)$ patients were asymptomatic. Indian studies have reported 30.8 to $38 \%$ patients to be asymptomatic which is similar to ours. Western studies have reported 47.7 to $64 \%$ patients to be asymptomatic which is higher than our study.

In our study $46(65.71 \%)$ patients had symptoms of liver disease. Right upper abdominal pain or discomfort $(55.71 \%)$, Fatigue $(52.85 \%)$ and malaise $(24.28 \%)$ were the dominant symptoms. Amarapurkar et al[16] reported $69.23 \%$ symptomatic patients having right hypochondrial pain as the presenting complaint. In the study by Agarwal et al[17] 64\% patients were symptomatic and right upper quadrant pain, fatigue and malaise were the main symptoms.

Raised serum triglycerides, total cholesterol, LDL and VLDL were seen in $67.14 \%, 45.71 \%, 34.28 \%$ and $25.71 \%$ of cases respectively. Roli Agrawal et al[15] reported hypertriglyceridemia in $63.7 \%$, hypercholesterolemia in $50 \%-80 \%$ patients, elevated LDL in $25 \%$ of patients and elevated VLDL in $56.5 \%$ of patients.

In our study low HDL was seen in $62.85 \%$ cases. Roli Agrawal et al[15] reported low HDL in $45.16 \%$ of patients. In our study, serum total cholesterol, serum HDL, serum LDL and VLDL shows statistical significance with increasing grades of NAFLD $(P<0.05)$. Serum triglyceride shows no statistical significance with increasing grades of NAFLD $(P=0.05)$.

The pathogenesis of NAFLD has remained poorly understood since the earliest description of the disease. Much current thinking remains hypothetical, since the mechanism or mechanisms are still being worked out.

Differences in body-fat distribution or antioxidant systems, possibly in the context of a genetic predisposition, may be among the explanations. A net retention of lipids within hepatocytes, mostly in the form of triglycerides, is a prerequisite for the development of nonalcoholic fatty liver disease. The primary metabolic abnormalities leading to lipid accumulation is not well understood, but they could consist of alterations in the pathways of uptake, synthesis, degradation, or secretion in hepatic lipid metabolism resulting from insulin resistance. Insulin resistance is the most reproducible factor in the development of nonalcoholic fatty liver disease[18].

Liver biopsy is the gold standard for diagnosis of NAFLD. But because of its invasiveness, complication, painfulness and sampling error it is not feasible in every asymptomatic cases. In this aspect ultrsonography offers promising role to diagnose NAFLD which is supported by significantly increased lipid profile values in our study.

Ultrasonography can be used for the early detection of NAFLD. Sonographically diagnosed NAFLD patients showed statistically significant association with serum lipid profile except serum triglyceride. It may be possible to say that Ultrasound is the least expensive modality for detecting changes associated with NAFLD and minimizes the exposure of unnecessary, expensive, complicated and tedious investigation in these patients and asymptomatic cases.

\section{Conflict of interest statement}

We declare that we have no conflict of interest.

\section{Acknowledgements}

We are thankful to Department of Radiodiagnosis, G.R. 
medical College J.A. Group of Hospitals. This is a pilot study funded by the Department of Radiodiagnosis with approval by institutional ethics committee of G.R. Medical College, Gwalior, Madhya Pradesh, India with grant no.-GRM/01/15/2009.

\section{Comments}

\section{Background}

NAFLD has emerged as the most common liver disease in the Western countries and now emerging in Asian countries. This is due to change in modifiable risk factors and metabolic syndrome. It is also increasing among Type-II DM. NAFLD appears to be common in some ethnic groups like Philipinos, Indians and aboriginals of Australia/Malaysia and have been proposed to be an etiological factor for hepatocellular carcinoma. The present study was designed to check the extent of dyslipidemia through non-invasive procedure at different grading of NAFLD replacing the traditional liver biopsy, which is painful and evasive procedure and sometimes can be life threatening.

\section{Research frontiers}

The new methodology of using ultrasonography replacing traditional biopsy is applied and this would be more comfortable for patients to grade the liver damage in NAFLD.

\section{Innovations and breakthroughs}

Application of USG in diagnosis of NAFLD and its correlation with extent of dyslipidemia at various stages of disease progression.

\section{Applications}

Biopsy can be replaced by ultrasonography. Liver biopsy is potentially life threatening complications like bleeding and is prone to sampling errors. But ultrasonography is a noninvasive and simple tool, it can be used for the early detection of NAFLD in asymptomatic patients in India.

\section{Peer review}

Over all the paper is worth publishing. It is short and descriptive and written well to attract readers' attention. The use of ultrasonography is clinically and financially practical for the early diagnosis of patients with NAFLD.

\section{References}

[1] Ludwig J, Viggiano TR, McGill DB, Oh BJ. Nonalcoholic steatohepatitis Mayo Clinic experiences with a hitherto unnamed disease. Mayo Clin Proc 1980; 55: 434-438.

[2] Pierre Bedossa. Current histological classification of non alcoholic fatty liver disease: strength and limitations. Hepatol Int 2013; 7: 1-6.

[3] David AS, Chang P, Chopra K. Non-alcoholic fatty liver disease: clinical review. Digest Dis Sci 2005; 50: 171-180.

[4] Agarwal AK, Jain V, Singla S, Baruah BP, Arya V, Yadav R, et al. Prevalence of non-alcoholic fatty liver disease and its correlation with coronary risk factors in patients with Type 2 diabetes. $J$ Assoc Physician India 2011; 59: 1-4.

[5] Bellentani S, Scaglioni F, Marino M, Bedogni G. Epidemiology of non-alcoholic fatty disease. Dig Dis 2010; 28(1): 155-161.

[6] Whye LC, Ping YL, Ching TC, Hamid JM, Siong LW. Prevalence of ultrasound diagnosed nonalcoholic fatty liver disease among rural indigenous community of sarawak and its association with biochemical and anthropometric measures. Southeast Asian J Trop Med Public Health 2013; 44(2): 309-317.

[7] Baffy G, Brunt EM, Caldwell SH. Hepatocellular carcinoma in nonalcoholic fatty liver disease: an emerging menace. J Hepatol 2012; 56: 1384-1391.

[8] De Minicis S, Marzioni M, Saccomanno S, Rychlicki C, Agostinelli L, Trozzi L, et al. Cellular and molecular mechanisms of hepatic fibrogenesis leading to liver cancer. Transl Gastrointest Cancer 2012; 1: 88-94.

[9] Obika M, Noguchi H. Diagnosis and evaluation of nonalcoholic fatty liver disease. Exp Diabetes Res 2012; 2012: 1-12.

[10] Chalasani N, Younossi Z, Lavine JE, Diehl AM, Brunt EM, Cusi K, et al. The diagnosis and management of non-alcoholic fatty liver disease: practice Guideline by the American Association for the Study of Liver Diseases, American College of Gastroenterology, and the American Gastroenterological Association. Hepatology 2012; 55: 2005-2023.

[11] Cadranel JF, Rufat P, Degos F. Practices of liver biopsy in France: results of a prospective nationwide survey. For the Group of Epidemiology of the French Association for the Study of the Liver (AFEF). Hepatology 2000; 32: 477-481.

[12] Figen CC, Nevil A, Hasan N. Complications and efficiency of liver biopsies using the Tru-Cut biopsy gun. J Infect Dev Ctries 2010; 4: 91-95.

[13] Shahin M, Rasoul S, Mehdi N, Masoumeh PH, Sadaf GS, Reza M, et al. Sampling error in histopathology findings of nonalcoholic fatty liver disease: a post mortem liver histology study. Arch Iran Med 2012; 15: 418-421.

[14] Ratziu V, Charlotte F, Heurtier A, Gombert S, Giral P, Bruckert E, et al. Sampling variability of liver biopsy in nonalcoholic fatty liver disease. Gastroenterology 2005; 128: 1898-1906.

[15] Agrawal R, Mishra S, Dixit VK, Rai S. Association of non-alcoholic fatty liver disorder with obesity. Indian J Prev Soc Med 2009; 40: 126-129.

[16] Amarapurkar DN, Amarapurkar AD. Nonalocholic steatohepatitis: clinicopathologic profile. J Assoc Physicians India 2000; 48: 311313.

[17] Agarwal SR, Malhotra V, Sakhuja P, Sarin SK. Clinical, biochemical and histological profile of nonacoholic steatohepatitis. Indian J Gastroenterol 2001; 20: 183-186.

[18] El-Koofy NM, Anwar GM, El-Raziky MS, El-Hennawy AM, ElMougy FM, El-Karaksy HM, et al. The association of metabolic syndrome, insulin resistance and non-alcoholic fatty liver disease in overweight/obese children. Saudi J Gastroenterol 2012; 18: 44-49. 